\title{
Satisfação do doente: Importância da comunicação médico-doente
}

Catarina Agostinho,* Miguel Cabanelas, ${ }^{*}$ Daniela Franco, ${ }^{*}$ Joana Jesus, * Henrique Martins**

\section{RESUMO}

Objectivos: Explorar aspectos da comunicação verbal e não verbal integrantes da relação médico-doente, determinando de que forma influenciam a satisfação dos doentes.

Tipo de Estudo: Transversal

Local: Serviço de Urgência (S.U.) do Centro Hospitalar Cova da Beira (CHCB).

População: Utentes do serviço de Urgência

Métodos: Aplicação de questionário por entrevista directa a 200 doentes com idade igual ou superior a 18 anos incidindo nas variáveis: género, idade e nível de instrução dos doentes e importância por estes atribuída a aspectos do médico como género, idade e aspectos da comunicação verbal (volume e velocidade do discurso, tipo de vocabulário, pronúncia) e não verbal (contacto visual, vestuário, penteado, proximidade física e gestos) deste.

Resultados: A maioria dos doentes refere atribuir importância à velocidade do discurso (83,5\%), ao contacto visual (90\%), à proximidade física $(68,5 \%)$ e ao facto de o médico acenar enquanto os ouve $(70 \%)$. Porém a pronúncia (68\%), a forma como o médico se veste $(69,5 \%)$ e se penteia $(78 \%)$ e o acto de gesticular $(63,5 \%)$ não parecem ser importantes. Quando inquiridos sobre aspectos mais específicos da comunicação, alguns doentes estão mais satisfeitos quando: o médico fala devagar (95,7\%), usa um tom igual ao seu (87\%), utiliza termos comuns $(94,3 \%)$, se veste de maneira informal $(82,3 \%)$, se senta à sua frente do outro lado da secretária (57,3\%), estabelece contacto físico $(67,5 \%)$ e quando os médicos do género feminino têm cabelo comprido $(66,7 \%)$ e cuidado $(93,8 \%)$. Ao serem questionados quanto ao género e idade do médico que mais contribui para a sua satisfação, os doentes revelam uma preferência por médicos do sexo feminino $(75,7 \%)$ e por aqueles que tenham idade inferior a 50 anos $(70,1 \%)$.

Conclusão: Parecem existir variáveis de comunicação verbal e não-verbal integrantes da relação médico-doente que influenciam a satisfação do doente.

Palavras-Chave: Comunicação Verbal; Comunicação Não-Verbal; Satisfação do Doente; Relação Médico-Doente.

\section{INTRODUÇÃO}

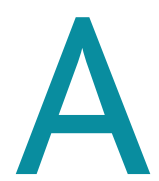
relação médico-doente, tão importante no contexto clínico, consiste na «Relação terapêutica baseada na confiança, honestidade, respeito e desejo mútuo que visa melhorar os ganhos em saúde». ${ }^{1}$

Actualmente, o doente é cada vez mais crítico relativamente à qualidade da relação estabelecida com o médico. O papel do doente nos cuidados que lhe são prestados tem-se modificado no tempo. Este passou de

*Aluno $5 .^{\circ}$ ano Mestrado Integrado em Medicina da Universidade da Beira Interior **Professor Auxiliar Convidado Faculdade Ciências da Saúde da Universidade da Beira Interior mero espectador para participante activo, cuja opinião deve ser valorizada. ${ }^{1}$ Se no passado as qualidades médicas eram apenas medidas pelo sucesso terapêutico e técnico, hoje é dada cada vez mais importância aos aspectos da comunicação na relação médico-doente. ${ }^{2,3}$

Estas percepções podem ter implicações clínicas uma vez que os doentes estão mais dispostos a seguir o aconselhamento médico, quando detectam por parte deste uma atitude calorosa, amistosa e preocupada com o seu bem-estar. ${ }^{4}$ Por outro lado, os doentes estão mais propensos a demonstrar indiferença quanto ao cumprimento terapêutico se o profissional de saúde adoptar uma atitude autoritária e a sua opinião não for 
tida em conta na tomada de decisões. ${ }^{4}$ Sendo assim, a percepção da qualidade do contacto médico-doente é determinante para a adesão terapêutica e para a adopção de comportamentos geradores de saúde. ${ }^{3}$

O objectivo desta investigação é explorar a importância de determinados aspectos da comunicação médico-doente, e avaliar a forma como estes determinam a satisfação percebida pelos doentes.

\section{COMUNICAÇÃO}

A comunicação na relação médico-doente engloba vários aspectos, dos quais se destacam: os participantes e os seus papéis; os erros na comunicação; o ambiente e os mecanismos necessários à construção e manutenção de uma boa comunicação; os ganhos resultantes de uma comunicação óptima; e os componentes de uma comunicação efectiva. ${ }^{1}$ Estes últimos, dividem-se em verbais e não-verbais.

Comunicação verbal compreende o discurso, falado ou escrito, em que a mensagem transmitida é veiculada sob a forma de signos linguísticos. Como tal, esta depende de elementos como a construção frásica, o vocabulário e o conteúdo da mensagem..$^{5} \mathrm{O}$ vocabulário utilizado pelo médico e a sua compreensão pelo doente são fulcrais na adesão à terapêutica. ${ }^{6}$ Frequentemente, os médicos utilizam termos demasiados tecnicistas para a instrução, idade e nível cultural do doente, que ameaçam a compreensão da mensagem. . $^{7,89}$

Já a Comunicação não-verbal, constitui uma forma não discursiva da linguagem. Esta serve-se de múltiplos canais de propagação, dependendo sobretudo das capacidades visuais e hápticas dos intervenientes. Os seus principais veículos são o corpo, as suas características físicas e movimentos (principal transmissor destes códigos); o homem, a sua apresentação e adereços; e o espaço, ambiente ou distância física entre os indivíduos. ${ }^{10}$ Os códigos que são transmitidos por este tipo de comunicação são o contacto físico (como, onde, a quem), a proximidade ou proxémia (grau de proximidade estabelecida para a transmissão da mensagem), a orientação (posição dos indivíduos, uns em relação aos outros), os movimentos da cabeça (representativos de consentimento ou discórdia, por exemplo), a expressão facial (representação mímica de emoções através da face), os gestos ou quinese (especialmente das mãos e dos braços), a postura (que espelha a forma de estar), o contacto visual (a sua presença ou evitamento), a prosódia (aspectos não-verbais do discurso que afectam o significado da mensagem ou que informam sobre o orador, tais como a entoação, a acentuação, o tom, o volume, a velocidade, a pronúncia, etc.). ${ }^{10,11}$ Estes dois tipos de comunicação, verbal e não-verbal, tendem a surgir acompanhados, mas poderão ou não ser congruentes entre si. ${ }^{12}$

\section{MÉTODOS}

Aplicou-se um questionário por entrevista directa, que foi desenvolvido originalmente para este trabalho. A base da construção do questionário foi a escala psicométrica de cinco níveis de importância de Likert - desde não importante a muito importante. ${ }^{13}$ Este contemplou dois grupos de questões. Um grupo relativo aos dados pessoais do doente, com o objectivo de caracterizar a amostra e determinar a sua influência nas restantes variáveis (género, idade, nível de instrução). O outro, relativo à importância dada a determinados aspectos da comunicação verbal e não-verbal da relação médico-doente, que contribuem para uma maior ou menor satisfação do doente (género, idade, volume e velocidade do discurso, tipo de vocabulário, pronúncia, contacto visual, vestuário, penteado, proximidade física e gestos - acenar e gesticular) (Quadros II, III e IV). Entende-se por acenar o gesto afirmativo com a cabeça; por proximidade física o acto de o médico tocar, por exemplo, no braço do doente; por cabelo comprido aquele que ultrapassa o maxilar inferior; por vestuário informal, o uso de calças de ganga, t-shirt, ou sapatilhas; por tipo de vocabulário, termos técnicos/científi-

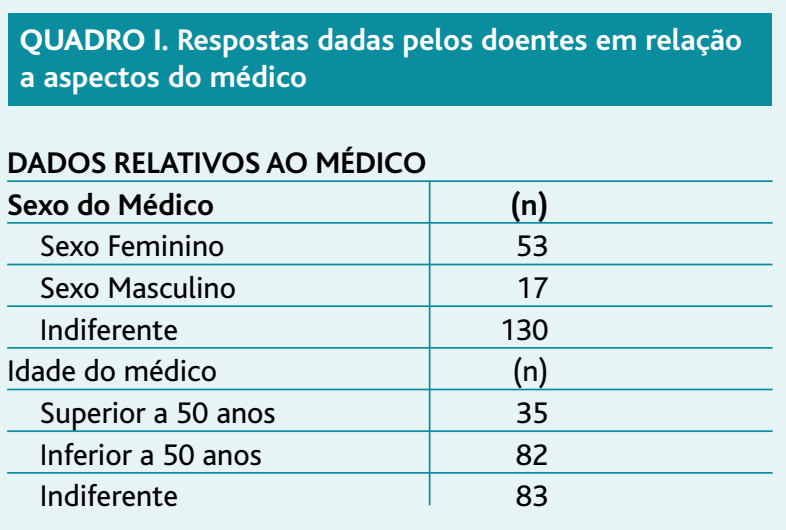




\begin{tabular}{|c|c|}
\hline \multicolumn{2}{|c|}{$\begin{array}{l}\text { QUADRO II. Respostas dadas pelos doentes em relação } \\
\text { a aspectos da comunicação verbal }\end{array}$} \\
\hline \multicolumn{2}{|l|}{ COMUNICAÇÃO VERBAL } \\
\hline Vocabulário & (n) \\
\hline Vocabulário (Importância) & 167 \\
\hline Termos comuns & 181 \\
\hline Termos científicos & 11 \\
\hline Indiferente & 8 \\
\hline
\end{tabular}

QUADRO III. Respostas dadas pelos doentes em relação a aspectos da comunicação verbal

\section{COMUNICAÇÃO NÃO-VERBAL}

\begin{tabular}{l|r|l|c}
\hline Penteado & (n) & Volume & (n) \\
\hline Penteado (Importância) & 44 & Volume (Importância) & 131 \\
\hline Médica Cabelo Curto & 16 & Tom igual & 168 \\
\hline Médica Cabelo Comprido & 32 & Tom diferente & 25 \\
\hline Indiferente & 152 & Indiferente & 7 \\
\hline Médica Cabelo Cuidado & 121 & Velocidade & (n) \\
\hline Médica Cabelo Descuidado & 8 & Velocidade (Importância) & 167 \\
\hline Indiferente & 71 & Falar depressa & 177 \\
\hline Médico Cabelo Curto & 85 & Falar devagar & 8 \\
\hline Médico Cabelo Comprido & 2 & Indiferente & 15 \\
\hline Indiferente & 113 & Pronúncia & (n) \\
\hline Médico Cabelo Cuidado & 129 & Pronúncia (Importância) & 64 \\
\hline Médico Cabelo Descuidado & 0 & Quinese & (n) \\
\hline Indiferente & 71 & Acenar (Importância) & 140 \\
\hline Vestuário & (n) & Gesticular (Importância) & 73 \\
\hline Vestuário (Importância) & 61 & Proximidade Física & (n) \\
\hline Formal & 17 & Proximidade Física (Importância) & 137 \\
\hline Informal & 79 & Senta ao lado & 65 \\
\hline Indiferente & 104 & Senta à secretária & 87 \\
\hline Contacto Físico & (n) & Indiferente & 48 \\
\hline Sem contacto & 54 & Contacto Visual & (n) \\
\hline Médico toca (por ex., braço) & 112 & Contacto Visual (Importância) & 180 \\
\hline Indiferente & 34 & & \\
\hline & & & \\
\hline
\end{tabular}

cos, por oposição a termos correntes/comuns.

O questionário foi aplicado a 200 doentes do Serviço de Urgência do Centro Hospitalar Cova da Beira, com idade igual ou superior a 18 anos, durante 10 dias, seleccionados aleatoriamente, dos meses de Novembro e Dezembro de 2008 (amostragem determinística por conveniência). Os doentes responderam ao questionário após a consulta de triagem de Manchester e antes da consulta médica, tendo sido solicitada a sua parti- cipação, a qual foi autorizada.

$\mathrm{Na}$ análise estatística analítica, para a determinação da significância estatística das relações entre as variáveis aplicou-se o teste de Qui-quadrado. ${ }^{14}$ Consideraram-se significativas as relações em que $p<0,05$. A análise foi realizada recorrendo às aplicações informáticas: Excel®, EpiInfo $®$ e Statcalc $®$.

\section{RESULTADOS}

Dos 200 doentes entrevistados no S.U. do CHCB: $59 \%(\mathrm{n}=118)$ do género feminino; $46,5 \%(n=93)$ tinha entre 18 e 44 anos, 35\% ( $n=70)$ (com) idade entre 45 e 64 anos e 18,5\% ( $n=$ 37) idade igual ou superior a 65 anos; $8,5 \%$ ( $n=17$ ) sem instrução ou que nunca completaram o $1^{\circ}$ ciclo, $30 \%$ (n $=60)$ com $1^{\circ}$ ciclo de instrução, $18,5 \%$ ( $\mathrm{n}=37$ ) com $2^{\circ}$ ou $3^{\circ}$ ciclo de instrução, $21 \%(n=42)$ com ensino secundário, $22 \%(n=44)$ com ensino superior.

\section{Satisfação do doente}

As respostas dadas pelos doentes em relação às variáveis estudadas da comunicação verbal e não-verbal constam dos Quadros II e III. No Quadro IV observam-se as relações entre os dados do doente e estas mesmas variáveis.

\section{DISCUSSÃO}

Importância atribuída à idade e à forma de vestir do médico

Neste estudo, os doentes inquiridos atribuem maior importância à idade $(58,5 \%)$ e à forma de vestir do médico (48\%) do que ao género deste (35\%). Estes resultados apoiam os obtidos por McNaughton-Filion et al. no seu estudo The physician's appearance. ${ }^{15}$

\section{Género}

Dos doentes que não responderam Indiferente, a maioria sente-se mais satisfeita ao ser atendida por um médico do género feminino (75,7\%) (Quadro I). Esta ten- 
QUADRO IV. Relação entre os dados do doente e as variáveis da comunicação verbal e não verbal; Teste Qui-Quadrado; Foram excluídas as respostas Indiferente.

\begin{tabular}{|c|c|c|c|c|c|c|c|c|c|}
\hline \multirow{3}{*}{ Dados do Médico } & \multicolumn{9}{|c|}{ Dados do Doente } \\
\hline & \multicolumn{2}{|c|}{ Género } & \multirow{2}{*}{$P$} & \multicolumn{2}{|c|}{ Instrução } & \multirow{2}{*}{$P$} & \multicolumn{2}{|c|}{ Idade } & \multirow{2}{*}{$P$} \\
\hline & Feminino & Masculino & & $\leq 9 .^{\circ}$ Ano & $>9 .^{\circ}$ Ano & & $\leq 65$ & $>65$ & \\
\hline \multicolumn{10}{|l|}{ Sexo } \\
\hline Feminino & 30 & 23 & \multirow{2}{*}{0,056} & 28 & 25 & \multirow{2}{*}{0,2081} & 41 & 12 & \multirow{2}{*}{0,1221} \\
\hline Masculino & 14 & 3 & & 6 & 11 & & 16 & 1 & \\
\hline \multicolumn{10}{|l|}{ Idade } \\
\hline$<50$ anos & 42 & 40 & \multirow{2}{*}{0,0831} & 37 & 45 & \multirow{2}{*}{0,00005} & 71 & 11 & \multirow{2}{*}{0,009} \\
\hline$\geq 50$ anos & 24 & 11 & & 30 & 5 & & 23 & 12 & \\
\hline \multicolumn{10}{|l|}{ Velocidade } \\
\hline Importância & 101 & 66 & 0,3387 & 99 & 68 & 0,0005 & 35 & 132 & 0,004 \\
\hline Falar devagar & 109 & 68 & \multirow{2}{*}{0,039} & 109 & 68 & \multirow{2}{*}{0,0005} & 144 & 33 & \multirow{2}{*}{0,01779} \\
\hline Falar depressa & 2 & 6 & & 0 & 8 & & 8 & 0 & \\
\hline \multicolumn{10}{|l|}{ Vocabulário } \\
\hline Importância & 101 & 66 & 0,339 & 98 & 69 & 0,279 & 138 & 29 & 0,910 \\
\hline Termos comuns & 112 & 69 & \multirow{2}{*}{0,023} & 108 & 73 & \multirow{2}{*}{0,128} & 148 & 33 & ( \\
\hline Termos científicos & 3 & 8 & & 4 & 7 & & 9 & 2 & 0,991 \\
\hline Contacto Visual & & & & & & & & & \\
\hline Importância & 102 & 78 & 0,04 & 109 & 71 & 0,023 & 35 & 145 & 0,003 \\
\hline Gesticular & & & & & & & & & \\
\hline Importância & 50 & 23 & 0,038 & 43 & 30 & 0,68 & 18 & 55 & 0,042 \\
\hline Acenar & & & & & & & & & \\
\hline Importância & 83 & 57 & 0,9 & 83 & 57 & 0,318 & 35 & 105 & 0,00002 \\
\hline Pronúncia & & & & & & & & & \\
\hline Importância & 40 & 24 & 0,489 & 42 & 22 & 0,091 & 18 & 46 & 0,006 \\
\hline Proximidade & & & & & & & & & \\
\hline Importância & 74 & 63 & 0,034 & 84 & 53 & 0,069 & 109 & 28 & 0,1068 \\
\hline Local onde o médic & se senta & & & & & & & & \\
\hline Atrás da secretária & 59 & 28 & 0052 & 47 & 40 & 0354 & 73 & 14 & 2787 \\
\hline Ao lado do doente & 34 & 31 & 0,032 & 40 & 25 & 0,354 & 50 & 15 & $0,218<$ \\
\hline Contacto físico & & & & & & & & & \\
\hline Sem contacto & 32 & 22 & 08808 & 20 & 34 & & 45 & 9 & 03076 \\
\hline Toca no braço & 65 & 47 & 0,0000 & 66 & 46 & 0,008 & 87 & 25 & 0,5910 \\
\hline Vestuário & & & & & & & & & \\
\hline Importância & 34 & 22 & 0,534 & 34 & 27 & 0,811 & 47 & 14 & 0,179 \\
\hline Formal & 5 & 12 & 0.049 & 15 & 2 & 0.0024 & 7 & 10 & \\
\hline Informal & 44 & 35 & 0,049 & 31 & 48 & $0,00<4$ & 72 & 7 & 0,000001 \\
\hline Médica - cabelo & & & & & & & & & \\
\hline Curto & 12 & 4 & 00009 & 6 & 10 & 08310 & 16 & 0 & 01396 \\
\hline Comprido & 8 & 24 & 0,0009 & 11 & 21 & 0,8310 & 28 & 4 & 0,1390 \\
\hline Cuidado & 69 & 52 & 06978 & 67 & 54 & 0002 & 96 & 25 & $0152 ?$ \\
\hline Descuidado & 4 & 4 & 0,0910 & 0 & 8 & 0,006 & 8 & 0 & $0,15<6$ \\
\hline
\end{tabular}


dência relativamente à preferência pelas médicas, já havia sido evidenciada noutro estudo por DiNicola e DiMatteo. Nesse estudo verificou-se que as médicas estabeleciam uma relação de maior cumplicidade com os doentes, utilizavam afirmações mais positivas e faziam um maior número de perguntas, levando a que os doentes se mostrassem mais comunicativos. ${ }^{4}$

Comparando as respostas dos doentes dos géneros feminino e masculino observou-se que a preferência por médicos do género feminino é mais evidente nos homens $(88,5 \%)$ do que nas mulheres $(68,2 \%)$ (Quadro IV). Porém, Derose et al. verificaram que o género do médico não se associa à satisfação dos doentes do sexo masculino. ${ }^{16}$

\section{Tipo de vestuário}

Dos doentes que não responderam «Indiferente», a maioria sente-se mais satisfeita quando os médicos se vestem de forma informal (82,3\%) (Quadro III). O estudo de McNaughton-Filion et al. apresentou resultados distintos, pois os doentes desse estudo preferiram para médicos do género masculino uma forma «tradicional» de vestir (bata branca, gravata, camisa e calças de fato). ${ }^{15}$ Comparando as respostas dos doentes tendo em conta o género e o nível de escolaridade, constatou-se que são as mulheres e os doentes com instrução superior à obrigatória que atribuem maior importância ao facto de o médico se vestir de forma informal $(89,8 \% \mathrm{e}$ 96\%, respectivamente) (Quadro IV).

\section{Importância atribuída à quinese}

Segundo Argyle, os gestos e os movimentos são importantes canais no processo comunicacional. ${ }^{10}$ No nosso estudo verificou-se que a maioria dos inquiridos atribui importância ao acenar por parte do médico (70\%), mas não (atribui) à gesticulação (63,5\%) (Quadro III). Porém, se atendermos à idade dos doentes verificou-se que os de idade superior 65 anos atribuem importância a este último aspecto (51,4\%) (Quadro IV).

\section{Importância atribuída à proximidade física}

A maioria dos doentes atribui importância à proximidade física estabelecida entre o médico e o doente (68,5\%), (Quadro III). Comparando as respostas dos doentes dos géneros feminino e masculino observou-se que, enquanto a maior parte dos homens sente-se mais satisfeita quando o(a) médico(a) se senta ao seu lado durante a consulta (52,5\%), a maior parte das mulheres prefere que o médico se sente à sua frente atrás da secretária $(63,4 \%)$. Analisando a variável instrução dos doentes, verificou-se que $76,7 \%$ daqueles com escolaridade igual ou inferior à obrigatória sentem-se mais satisfeitos quando o médico lhes toca (por exemplo, no braço), enquanto apenas $57,5 \%$ dos que têm escolaridade superior revelam esta preferência (Quadro IV).

\section{Importância atribuída à forma como o médico se} penteia

A maioria dos doentes não atribui importância à forma como o médico se penteia (78\%) (Quadro III), mas questionados sobre a sua preferência relativamente a aspectos específicos, a maior parte dos homens sente-se mais satisfeita se as médicas que os atendem tiverem cabelo comprido (85,7\%), enquanto a maior parte das mulheres (60\%) prefere o contrário. Os doentes com escolaridade igual ou inferior ao $9 .^{\circ}$ ano mostram-se mais satisfeitos quando atendidos por médicas com penteado cuidado (100\%) (Quadro IV).

\section{Tipo de vocabulário}

Williams et al. no seu artigo de revisão The Role of Health Literacy in Patient-Physician Communication refere que múltiplos estudos documentam que a iliteracia dos doentes e os termos científicos usados pelo médico resultam numa comunicação confusa e inadequada, levando a que os doentes não compreendam nem a sua doença nem as opções terapêuticas. ${ }^{9,17}$ No nosso estudo $90,5 \%$ dos doentes preferem que o médico utilize termos comuns. Porém ao analisar a relação entre o nível de escolaridade do doente e a sua preferência por termos comuns, não se obteve significância estatística.

\section{Idade}

Dos que não responderam «Indiferente», a maioria dos doentes prefere ser atendida por médicos com idade inferior a 50 anos $(70,1 \%$ ) (Quadro I) o que apoia McNaughton-Filion et al. quando este refere no seu estudo que os doentes preferem ser atendidos por médicos com idade compreendida entre os 30 e os 50 anos. ${ }^{15}$ Atendendo à variável instrução do doente, verificou-se que a maioria das pessoas com instrução superior à 
obrigatória sente-se mais satisfeita ao ser atendida por médicos com idade inferior a 50 anos (90\%), não sendo esta preferência tão evidente naquelas com escolaridade inferior $(55,2 \%)$. Relacionando as variáveis idade do doente e idade do médico verificou-se que enquanto os doentes com idade igual ou inferior a 65 anos (75\%) preferem ser atendidos por médicos com idade inferior a 50 anos, os doentes com idade superior a 65 anos preferem ser atendidos por médicos com idade superior a 50 anos $(52,1 \%)$ (Tabela IV).

\section{Contacto visual}

Nunes, no seu livro Comunicação em Contexto Clínico, defende que o contacto visual que o médico estabelece com o doente constitui uma das principais «regras de boa escuta», uma vez que demonstra interesse pelo doente e pela sua mensagem. ${ }^{18}$ Tal é reforçado pelo nosso estudo, já que a maioria dos doentes inquiridos referiu sentir-se mais satisfeita quando é estabelecido contacto visual (90\%). Tendo em conta a instrução dos doentes, constatou-se que aqueles com escolaridade inferior são os que atribuem importância a este aspecto de forma mais evidente $(95,6 \%)$ (Tabelas III e IV).

\section{Velocidade do discurso}

Nunes enfatiza ainda o ritmo e a dicção do diálogo, referindo que é «muito importante a velocidade com que se dizem as coisas». O autor diz ser fundamental a adequação da velocidade com que o médico dá a informação à velocidade com que o doente fala. Estas conclusões apoiam os resultados do nosso estudo, já que se aferiu que a maioria dos doentes considera importante para a sua satisfação a velocidade do discurso do médico (83,5\%). Por outro lado, apesar da maioria dos doentes inquiridos não atribuir importância à pronúncia do médico (68\%), 51,4\% dos doentes com idade superior a 65 anos considera importante esta variável (Tabela III).

\section{IMPLICAÇÕES}

«O remédio mais utilizado em medicina é o próprio médico». ${ }^{19}$ Esta afirmação de Turini et al. demonstra a enorme importância da relação médico-doente e do processo comunicacional que a veicula. Como tal, torna-se fundamental para uma boa prática clínica que o médico avalie as suas competências comunicacionais, de forma a poder trabalhá-las e adequá-las às diversas situações e desafios que lhe são propostos. Se tal exercício não for realizado, o profissional poderá manter indefinidamente um estilo de comunicação inadequado que dificultará a sua relação com os seus doentes. ${ }^{6}$ Este efeito terapêutico da relação médico-doente tem vindo a ganhar maior importância à medida que surgem no dia-a-dia cada vez mais questões sociais, que ultrapassam os conhecimentos puramente biomédicos. Ong et al. relata que a comunicação entre o médico e o doente pode repercutir-se na satisfação, adesão e compreensão da mensagem por parte do doente. ${ }^{19}$ Nesta perspectiva, torna-se cada vez mais emergente a necessidade de se incluir «A Comunicação» na formação do médico. ${ }^{20}$

Muitas escolas médicas norte-americanas e europeias já se preocupam com a temática da relação médico-doente e com o ensino de capacidades fundamentais para a comunicação, apresentando programas curriculares estruturados e específicos. ${ }^{21}$ Em Portugal, há também essa preocupação por parte das Faculdades Médicas, dada a extraordinária importância destes conceitos para a formação dos seus alunos. Uma proposta de ensino que os contemple privilegia a formação humana e ética dos futuros médicos, o que, juntamente com a aquisição das competências biomédicas, os torna profissionais mais atentos, completos e competentes. ${ }^{20}$ Com esta consciência, cabe agora às Faculdades Portuguesas manter o investimento nesta área, quer ao nível de investigação quer de ensino, de forma a dotar os seus alunos de plenas capacidades relacionais.

\section{LIMITAÇÕES}

O tamanho da amostra constitui uma limitação. Uma amostra maior poderia permitir outra análise.

A condição de saúde dos doentes no contexto de urgência é outra limitação, dado que muitos mostraramse pouco receptivos e disponíveis para colaborar.

O questionário elaborado para este estudo pode constituir uma limitação importante pela sua extensão, devido ao número de variáveis em estudo.

A análise isolada das variáveis comporta restrições, uma vez que deverão ser contextualizadas na singularidade da relação médico-doente estabelecida.

Estudar a população abrangida pelo CHCB, população essa com características próprias, pode condicio- 


\section{nar os resultados obtidos, limitando a extrapolação des-} tes para a população nacional.

\section{REFERÊNCIAS BIBLIOGRÁFICAS}

1. Defining the Patient-Physician Relationship for the 21st Century. 3rd Annual Disease Management Outcomes Summit; 2003 Oct 30 - Nov 2; Phoenix, Arizona. Nashville (TN): American Healthways; 2003.

2. Hespanhol A, Vieira A, Pereira AC. Monitorização da satisfação dos utentes do Centro de Saúde São João (2007 e comparação com 2002 a 2006). Rev Port Clin Geral 2008 Mai-Jun;24 (3): 363-72.

3. Haddad S, Potvin L, Roberge D, Pineault R, Remondin M. Patient perception of quality following a visit to a doctor in a primary care unit. Fam Pract 2000 Feb; 17 (1): 21-9.

4. Brannon L, Feist J. Psicología de la salud. Madrid: Thompson Learning; 2000. p.123-8.

5. Rodrigues IG. Comunicação intercultural. Disponível em: http://web.letras.up.pt/irodrig/comunica\%C3\%A7\%C3\%A30\%20intercultural-cultura.ppt [acedido em 10/10/2008]

6. Silva PR. A comunicação na prática médica: seu papel como componente terapêutico. Rev Port Clin Geral 2008 Jul-Ago; 24 (4): 505-12.

7. Vaysse J. Culture and communication in medicine. Symposium Family and Culture in General Practice - The European Approach; 1994 May 25-28; Lisboa, Portugal. Paris; 1994.

8. Baylon C, Mignot X. La communication. Paris: Nathan Université; 1991.

9. Almeida $H$, Versiani $E$, Dias $A$, Novaes $M$, Trindade E. Adesão a tratamentos entre idosos. Com. Ciências Saúde 2007;18 (1): 57-67.

10. Mesquita RM. Comunicação não-verbal: relevância na atuação profissional. Rev Paul Educ Fis 1997 Jul-Dez; 11 (2):155-63.

11. Ribeiro JL. Psicologia e Saúde. Lisboa: ISPA; 1998. p. 96-77.

12. Comunicação não verbal. Disponível em http://www.addinteractive. com/add2us/add2us04/add2us04_com_nverbal.pdf [acedido em 10/10/2008].

13. Likert scale. Disponível em: http://en.wikipedia.org/wiki/Likert_scale [acedido em 18/01/2009].

14. Pina AP. Investigação e Estatística com o Epilnfo. Disponível em: http://www.saudepublica.web.pt/03-Investigacao/032-EpilnfoSoftware/apresenta\%C3\%A7\%C3\%A3o_epiinfo_2000.htm [acedido em 31/12/2008].
15. McNaughton-Filion L, Chen JS, Norton PG. The physician's appearance. Fam Med 1991 Mar-Apr; 23 (3): 208-11.

16. Derose KP, Hays RD, McCaffrey DF, Baker DW. Does physician gender affect satisfaction of men and women visiting emergency department? J Gen Intern Med 2001 Apr; 16 (4): 218-26.

17. Williams MV, Davis T, Parker RM, Weiss BD. The role of health literacy in patient-physician communication. Fam Med 2002 May; 34 (5): 3839.

18. Nunes JM. Comunicação em contexto clínico. Lisboa: Edições Bayer Health Care; 2007.

19. Turini B, Neto DM, Tavares MS, Nunes SOV, Silva VRLM, Thompson Z. Comunicação no ensino médico: estruturação, experiência e desafios em novos currículos médicos. Rev Bras Educ Med 2008 jun; 32 (2): 26470

20. Sucupira AC. A importância do ensino da relação médico-paciente e das habilidades da comunicação na formação do profissional de saúde. Interface - Comunic Saúde Educ 2007 set-dez;11 (23): 619-35.

21. Rossi PS, Batista NA. O ensino da comunicação na graduação em medicina - uma abordagem. Interface - Comunic Saúde Educ 2006 janjun;10 (19): 93-102.

22. Rodrigues L. Psicologia $12^{\circ}$ ano. $2^{\mathrm{a}}$ ed. Lisboa: Plátano Editora; 2001. p. 546-7.

23. Monteiro MM, Pereira N. Psicologia $12^{\circ}$ Ano Acesso Ensino Superior 2004. Porto: Porto Editora; 2004.

24. Aranha ML, Martins MH. Filosofando: Introdução à Filosofia. São Paulo: Editora Moderna; 1995.

25. Hoffman B. The Encyclopedia of Educational Technology. Disponível em: http://coe.sdsu.edu/eet/Articles/surveyquest/index.htm [acedido em 10/01/2009].

26. Neto F. Psicologia Social. $1^{\circ}$ vol. Lisboa: Universidade Aberta; 1998. p. 501-2, 507, 546-51.

\section{ENDEREÇO PARA CORRESPONDÊNCIA}

Daniela Franco

E-mail: comunicacao.medico.doente@gmail.com

Recebido em 02/07/2010

Aceite para publicação em 26/02/2010 


\section{ABSTRACT}

\section{PATIENT'S SATISFACTION: THE IMPORTANCE OF THE PATIENT-PHYSICIAN COMMUNICATION}

Objectives: To evaluate and understand which aspects of verbal and non-verbal communication patients refer as more satisfying.

Type of Study: Cross-sectional Study

Location: Centro Hospitalar Cova da Beira (CHCB) - Emergency Room

Study Population: Patients attending the Emergency Room

Methods: A questionnaire was applied by direct interview to 200 patients with 18 years-old or older, at the Emergency Room, during 10 random days, between November and December 2008.

Results: Most participants referred that speed of speech (83.5\%), visual contact (90\%), physical closeness (68,5\%) and doctor's movements, such as nodding while listening patient's complains (70\%), are important for their satisfaction. On the other hand, they believe that doctor's hair (78\%), way of dressing $(69,5 \%)$ and accent $(68 \%)$ are irrelevant. When asked about more specific aspects of communication, they say they are more satisfied when the doctor speaks slowly $(95,7 \%)$, as loud as them $(87 \%)$ with common expressions $(94,3 \%)$, dresses up in an informal manner $(82,3 \%)$, touches them $(67,5 \%)$, sits in front of them behind the desk $(57,2 \%)$, and when female doctors have long $(66,7 \%)$ and well combed hair $(93,8 \%)$. When asked about the sex and age of the physician who contributes most to their satisfaction, patients show a preference for female physicians (75,7\%); and by those who are under 50 years-old $(70,1 \%)$.

Conclusion: There are variables of verbal and non-verbal communication part of the doctor-patient relationship that influence the patient s satisfaction.

Key-Words: Verbal Communication; Non-Verbal Communication; Patient's Satisfaction; Patient-Physician Relationship. 